\title{
Stent Fitting using Cylindrical Affine Transformation Model for Pulmonary Artery
}

\author{
${ }^{1}$ Himani Arora \\ ${ }^{1}$ K.K.Shukla \\ ${ }^{2}$ Luc Duong \\ ${ }^{1}$ Department of Computer Engineering, Indian Institute of Technology (BHU), Varanasi, India \\ ${ }^{2}$ Department of Software and IT Engineering, Ecole de technologie superieure (ETS), Montreal, Canada
}

\begin{abstract}
The stents are becoming widely used as percutaneous interventions of pulmonary artery are progressively replacing conventional open heart surgeries. Presently, X-ray images of pulmonary artery i.e. angiograms are visually inspected by physician to determine the proper stent size. The size estimation of stent done preoperative which has not been assessed so far, is studied in this paper. A reliable method to determine the size of stent using a deformation model before performing angioplasty is proposed. A cylindrical affine transformation model is used to predict the expected deformation of the stent to fit it as closely as possible into artery. The method was evaluated on artery data set of 5 cases in which the patient was suffering from stenosis of pulmonary artery to obtain satisfactory results.
\end{abstract}

\section{Keywords}

Percutaneous interventions, Stent, Fitting, Pulmonary artery, Cylindrical Affine Transformation, Stenosis

\section{INTRODUCTION}

Non-operative or interventional procedure for treatment of vascular structures suffering from intravascular or intracardiac stenosis is recommended over conventional surgical procedures [1]. As described by Dotter and Judkins, this procedure requires the vessel to be held open [2], stent implantation exactly serves that purpose. Stents exert radial force on vessel walls preventing their acute closure thereby improving the blood flow [3]. The main reason for success of stent implantation in pulmonary artery in spite of adverse risk factors involved is same as found out in trails initiated by Serruys et al for coronary artery i.e. reduced angiographic restenosis rates and improvement in the patient clinical output as compared to balloon angioplasty as proved [4][5][6][7].

Stents are widely used for angioplasty but there is no tool/means available to judge the size of the stent which is appropriate for given pulmonary artery. In absence of reliable method to estimate the exact size of the stent, outcome is determined by physician experience [8]. Deployment of the stent which is too large into an artery can damage it causing arterial dissection or even rupture in worse cases. On the other hand, implanting a smaller stent will require re-operation to introduce larger stent [9][10]. This problem becomes acute in case of pediatric cases as lumen of the artery in these cases in very small in comparison to adults. So, there is need to especially manufacture the stent, making the determination of stent size even more important.

Introduction of stent locally increase the curvature of artery resulting in low and high shear stress regions near stent edges which can cause in stent re-stenosis [11]. Since shape of the stent is responsible for causing re-stenosis, the possible solution can be deforming the stent to make it fit the curvature of artery as closely as possible.

The method similar to Quantitative Coronary Analysis (QCA) systems can be deployed for pulmonary artery to estimate the width (chord length) of the artery at location of stenosis [12], which can further be used to estimate stent size. According to some cardiologists appropriate stent size can be approximated by adding the chord length of an artery just before the blockage and the chord length just after the point where stenosis ends, then taking their average and multiplying by $90 \%$ [9]. However, determination of proper stent size from QCA system is not sufficient. Unfortunately owing to complex artery tree structure and lack of precision in determination of chord length through QCA system this method of stent size estimation can prove very unreliable.

Therefore, an approach that captures a high level information about a pulmonary artery and stent and use it to output a metric that defines how appropriate is the given stent size when fitted into the pulmonary artery done preoperative would be valuable to the cardiologist. A novel method for automatic matching of the stent to the artery points followed by subsequent deformation of stent is proposed. A 3D model of artery was obtained by segmenting CT volumetric data set of pulmonary artery using TurtleSeg software [13][14]. This model along with 3D model of stent was used to simulate stent deformation for perfect fitting and determine parameters of Cylindrical Affine Transformation model to mathematically express the deformation. The proposed method was evaluated on artery dataset of 5 patients and satisfactory results were obtained.

In the next section, some mathematical preliminaries utilized in this paper are introduced. While the stent to artery fitting algorithm is discussed in section 3, the experiment and simulated results obtained from different artery data set are considered in section 4 . The section 5 draws conclusion from the work done so far and discusses some future work.

\section{MATHEMATICAL PRELIMINARIES}

\subsection{Cylindrical Affine Transformation Model}

A parametric transformation model is needed to predict the expected deformation of the stent to perfectly fit artery in terms of its parameters. With the stent and artery being cylindrical in shape, the use of a Cylindrical Affine Transformation (CAT) model is proposed to simulate its deformation.

This model is compact and easy to implement. Using centre of the cylinder $\left(\mathrm{x}_{0}, \mathrm{y}_{0}, \mathrm{z}_{0}\right)$, cylindrical scaling $\left(\mathrm{s}_{1}, \mathrm{~s}_{2}, \mathrm{~s}_{3}\right)$, cylindrical shearing $\left(\lambda_{1}, \lambda_{2}\right)$, cylindrical centre $\left(\theta_{0}, r_{0}\right)$ and the Cartesian translation $\left(\mathrm{t}_{\mathrm{x}}, \mathrm{t}_{\mathrm{y}}, \mathrm{t}_{\mathrm{z}}\right)$ as parameters of CAT model, The following equations are used [15]: 
$=\sqrt{\left(x-x_{0}\right)^{2}+\left(y-y_{0}\right)^{2}}$

$\theta$

$=\operatorname{atan}\left[\frac{\left(y-y_{0}\right)}{\left(x-x_{0}\right)}\right]$

$\hat{\theta}$

$=\mathrm{s}_{1}\left(\theta-\theta_{0}\right)+\lambda_{1}$

$\hat{r}=\lambda_{2}\left(\theta-\theta_{0}\right)+s_{2}\left(r-r_{0}\right)$

$+r_{0}$

$\hat{x}$

$=\left(\lambda_{2}\left(\theta-\theta_{0}\right)+s_{2}\left(r-r_{0}\right)\right.$

$\left.+\mathrm{r}_{0}\right) \cos \left[\mathrm{s}_{1}\left(\theta-\theta_{0}\right)+\lambda_{1}\left(\mathrm{r}-\mathrm{r}_{0}\right)+\theta_{0}\right]+\mathrm{x}_{0}$

$+\mathrm{t}_{\mathrm{x}}$

$\hat{y}=\left(\lambda_{2}\left(\theta-\theta_{0}\right)+s_{2}\left(r-r_{0}\right)\right.$

$\left.+r_{0}\right) \sin \left[s_{1}\left(\theta-\theta_{0}\right)+\lambda_{1}\left(r-r_{0}\right)+\theta_{0}\right]$

$+y_{0}$

$+t_{y}$

$\hat{\mathrm{Z}}$

$=\mathrm{s}_{3}\left(\mathrm{z}-\mathrm{z}_{0}\right)+\mathrm{z}_{0}+\mathrm{t}_{\mathrm{z}}$

\subsection{Distance metrics}

(i) Euclidean Distance: It is most commonly used distance metric. Mathematically, it is simply the geometric distance between two points. In Cartesian coordinate, if $\mathbf{x}_{1}=\left(\mathrm{x}_{1}, \mathrm{y}_{1}, \mathrm{z}_{1}\right)$ and $\mathbf{x}_{2}=\left(\mathrm{x}_{2}, \mathrm{y}_{2}, \mathrm{z}_{2}\right)$ are two points in three dimensional space, then the Euclidean distance between them is given by:

$\mathrm{E}\left(\mathbf{x}_{1}, \mathbf{x}_{2}\right)$

$=\sqrt{\left(x_{1}-x_{2}\right)^{2}+\left(y_{1}-y_{2}\right)^{2}+\left(z_{1}-z_{2}\right)^{2}}$

(ii) Shortest Distance between a point and a line in three dimensions: The shortest distance (perpendicular) of a point $\mathbf{x}_{\mathbf{0}}$ from a line in three dimensional space specified by two points $\mathbf{x}_{1}=\left(x_{1}, y_{1}, z_{1}\right)$ and $\mathbf{x}_{2}=\left(x_{2}, y_{2}, z_{2}\right)$ is given by (See Fig. 1) [16]:

$d$

$=\frac{E\left(\left(x_{0}-x_{1}\right) \times\left(x_{0}-x_{2}\right)\right)}{E\left(x_{2}-x_{1}\right)}$

\subsection{Other formulae}

(i) Coordinates of point where perpendicular from a point meets line in three dimensions: The coordinates of the point $\mathbf{x}_{\mathbf{n}}$ where perpendicular from a point $\mathbf{x}_{\mathbf{0}}$ meets a line in three dimensional space specified by two points $\mathbf{x}_{\mathbf{1}}=\left(\mathrm{x}_{1}, \mathrm{y}_{1}, \mathrm{z}_{1}\right)$ and $\mathbf{x}_{\mathbf{2}}=$ $\left(\mathrm{x}_{2}, \mathrm{y}_{2}, \mathrm{z}_{2}\right)$ is given by (See Fig. 1$)$ :

$\left(\mathrm{x}_{\mathrm{n}}, \mathrm{y}_{\mathrm{n}}, \mathrm{z}_{\mathrm{n}}\right)=\left(\mathrm{x}_{1}+\mathrm{t}\left(\mathrm{x}_{2}-\mathrm{x}_{1}\right), \mathrm{y}_{1}+\mathrm{t}\left(\mathrm{y}_{2}-\mathrm{y}_{1}\right), \mathrm{z}_{1}+\mathrm{t}\left(\mathrm{z}_{2}-\mathrm{z}_{1}\right)\right)$

(10)

Where $\mathrm{t}=\frac{\left(x_{0}-x_{1}\right)\left(x_{2}-x_{1}\right)+\left(y_{0}-y_{1}\right)\left(y_{2}-y_{1}\right)+\left(z_{0}-z_{1}\right)\left(z_{2}-z_{1}\right)}{\left(x_{2}-x_{1}\right)^{2}+\left(y_{2}-y_{1}\right)^{2}+\left(z_{2}-z_{1}\right)^{2}}$

(ii) Angles between two vectors: The angle between two vectors represented by $\mathrm{A}=\left(a_{1} \hat{\imath}, b_{1} \hat{\jmath}, c_{1} \hat{k}\right)$ and $\mathrm{B}=\left(a_{2} \hat{\imath}, b_{2} \hat{\jmath}, c_{2} \hat{k}\right)$ respectively is given by:

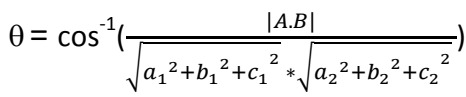

(11) (iii) Rotation about arbitrary axis: Let $A=(u \hat{\imath}, v \hat{\jmath}, w \hat{k})$ is a unit vector about which point $\mathrm{P}=(\mathrm{x}, \mathrm{y}, \mathrm{z})$ is to be rotated by angle $\theta$ considering $(\mathrm{a}, \mathrm{b}, \mathrm{c})$ as origin. Coordinates of point $\mathrm{P}$ after rotation is given by:

$$
\begin{array}{r}
x^{\prime}=\left(a\left(v^{2}+w^{2}\right)-u(b v+c w-u x-v y-w z)(1-\cos \theta)\right) \\
+x \cos \theta+(-c v+b w-w y+v z) \sin \theta \\
y^{\prime}=\left(b\left(u^{2}+w^{2}\right)-v(a u+c w-u x-v y-w z)(1-\cos \theta)\right) \\
+y \cos \theta+(c u-a w+w x-u z) \sin \theta \\
z^{\prime}=\left(c\left(u^{2}+v^{2}\right)-w(a u+b v-u x-v y-w z)(1-\cos \theta)\right) \\
+z \cos \theta+(-b u+a v-v x+u y) \sin \theta
\end{array}
$$

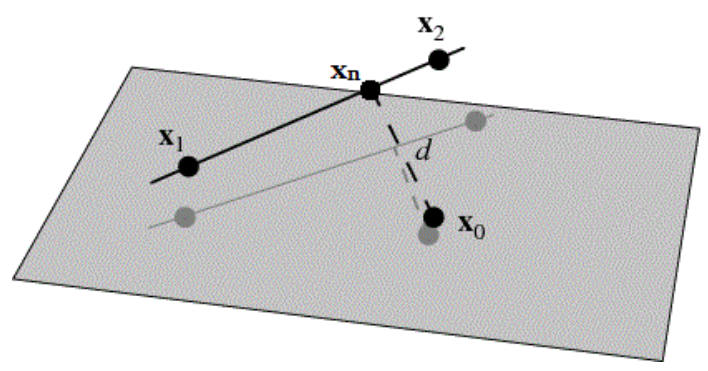

Fig. 1: Illustrating Shortest Distance between a point and a line in three dimensions [16]

\section{STENT TO ARTERY FITTING ALGORITHM}

The proposed method is implemented on 3D model of artery obtained by segmenting CT volumetric data set of pulmonary artery and a 3D stent model. The centerline interpolation was done followed by alignment of stent with respect to location of stenosis (determined subjectively by visual inspection). A mapping from stent to artery data set was thus obtained to complete the stent to artery fitting. Next, a deformation model was used to model deformation in terms of its parameters. Each step is described in detail below.

\section{Step 1: Interpolation of Centerline points using Spline}

A set of points serving as segmented centerline of the vessel was obtained from custom made software. In order to interpolate the data, the following 4 different approaches were used for interpolation:

- 'nearest' for nearest neighbor interpolation

- 'linear' for linear interpolation

- 'spline' for cubic spline interpolation

- 'cubic' for cubic interpolation

As interpolation using spline was found to be most effective, one dimension spline interpolation for each $\mathrm{x}, \mathrm{y}$ and $\mathrm{z}$ coordinates was used [17]. Approximately 200 points were generated between every two points of the centerline (See Fig. 2). 


\section{Step 2: Initial Alignment of stent}

By default, the stent was aligned along Y-axis, while the location and orientation of stenosis in artery changes with every data-set. In order to speed up the stent artery fitting process, stent was aligned using these three basic transformations:

\section{(1) Rotation:}

- A tangent is drawn to the centerline where stenosis has occurred. Let this vector be $\mathrm{T}$.

- A vector is considered along the stent axis. Let this vector be A.

- Angle between these vectors is calculated using Equation (11). Let that angle be called $\theta_{0}$.

- A perpendicular $\mathrm{P}$ is drawn to vector $\mathrm{A}$ and $\mathrm{T}$ by taking their cross product. Normalize the vector such that $\mathrm{P}$ is a unit vector.

- A is rotated by angle $\theta_{0}$ taking $\mathrm{P}$ as arbitrary axis and location of stenosis as origin using Equation (12).

(2) Scaling:

Scaling was done based on subjective evaluation to make sure that stent is entirely inside the artery and not protruding out. This is done to so that deformation of stent is in one direction.

\section{(3)Translation:}

This step involves bringing the stent to the point of stenosis. The stent is simply translated to the point on centerline where tangent was drawn in previous step.

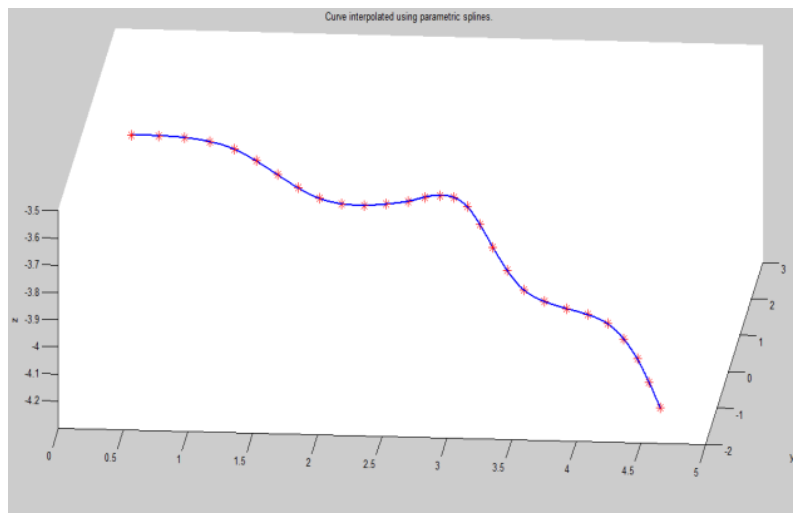

Fig. 2: Illustrating Interpolation of Centerline points of the pulmonary artery. Blue line indicates interpolated points while red * shows initial centerline points available to us.

\section{Step 3: Calculation of center for each stent point}

After the alignment of stent at desired location inside the artery, the center for each stent point on interpolated centerline was obtained using these steps:

(i) Consider a particular stent point, $\mathrm{S}$, whose projected center on interpolated centerline (obtained in step 1) is to be calculated.

(ii) The two points on interpolated centerline which are closest to $\mathrm{S}$ are obtained. Let them be called as A and B.

(iii) A perpendicular was drawn from $\mathrm{S}$ to line joining $\mathrm{A}$ and $\mathrm{B}$ as shown in Fig.3. The point of intersection $\mathrm{C}$ was obtained using equation (10).

(iv) Steps (i) to (iii) were repeated for other stent points and center $\mathrm{C}$ was calculated for each point.

\section{Step 4: Determining closest artery point}

This is the most important step of artery fitting. Let I indicate the set of artery points. Let $\mathrm{A}_{\mathrm{i}}$ and $\mathrm{S}$ represents a point from artery data set and stent data set respectively. Let $\mathrm{L}$ denotes line joining $\mathrm{S}$ and C (obtained in step 3).

$\mathrm{di}_{1}=$ Perpendicular distance between line $\mathrm{L}$ and point Ai using equation (9).

$\mathrm{di}_{2}=$ Euclidean distance between $\mathrm{S}$ and $\mathrm{A}_{\mathrm{i}}$ using equation (8).

$\mathrm{d}_{\min }=\underbrace{\min }_{i \in I}\left(\mathrm{di}_{1}+\mathrm{di}_{2}\right)$

Let the artery point in I that yields the minimum distance is represented by $\mathrm{A}_{\mathrm{c}}$

$A_{c}$ is calculated for each stent point. Fig. 3 clearly illustrates the relation between the stent point and closest artery point.

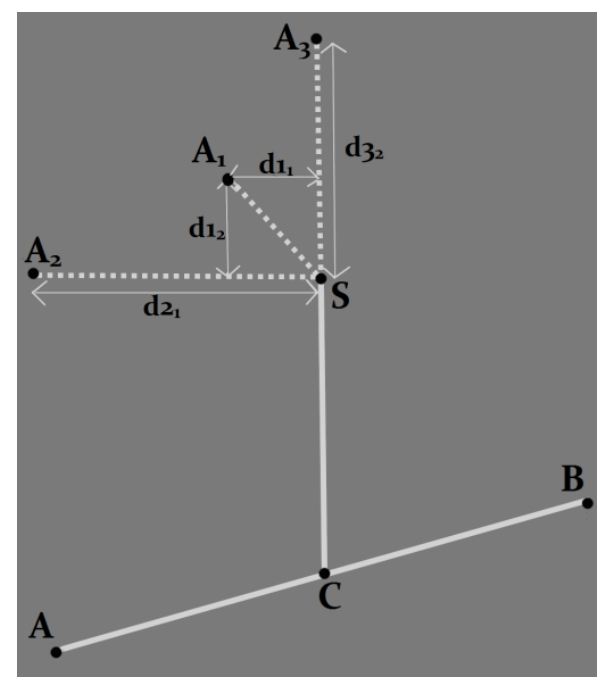

Fig.3: Relation between stent point $S$ and closest artery point

$A_{1}$

\section{Step 5: Deformation of stent}

After obtaining the closest artery point, each stent point $\mathrm{S}$ is deformed to coincide with its closest artery point $\mathrm{A}_{\mathrm{c}}$. This stentartery fitting was confirmed by visual simulation as discussed in next section.

\section{Step 6: Parameters of CAT Model.}

The stent has undergone deformation to perfectly fit the artery. This deformation is expressed mathematically using CAT model. Cylindrical scaling parameters $\left(\mathrm{s}_{1}, \mathrm{~s}_{2}, \mathrm{~s}_{3}\right)$ out of 13 parameters discussed in Section 2 were used to model this deformation. Cylindrical shearing parameters $\left(\lambda_{1}, \lambda_{2}\right)$, are assumed to be zero for sake of convenience, while centre of the cylinder $\left(\mathrm{x}_{0}, \mathrm{y}_{0}, \mathrm{z}_{0}\right)$ is set to be equal to center of stent point calculated in step 3. Cylindrical centre $\left(\theta_{0}, r_{0}\right)$ and the Cartesian translation $\left(t_{x}, t_{y}, t_{z}\right)$ parameters are zero due to alignment of stent in Step 2.

For each stent point $\mathrm{S}$ the original and deformed coordinates are expressed in cylindrical coordinates using equation (1) and (2). Let $\left(\mathrm{r}_{\mathrm{s}}, \theta_{\mathrm{s},} \mathrm{z}_{\mathrm{s}}\right)$ and $\left(\mathrm{r}_{\mathrm{s}}{ }^{\prime}, \theta_{\mathrm{s}}{ }^{\prime}, \mathrm{z}_{\mathrm{s}}{ }^{\prime}\right)$ represents the original and 
deformed cylindrical coordinates of stent point $\mathrm{S}$ respectively. Taking into consideration the constraints mentioned above and using equation (3), (4) and (7), $s_{1}, s_{2}, s_{3}$ were solved:

$$
\mathrm{s}_{1}=\mathrm{r}_{\mathrm{s}}{ }^{\prime} / \mathrm{r}_{\mathrm{s}} \quad \mathrm{s}_{2}=\theta_{\mathrm{s}}{ }^{\prime} / \theta_{\mathrm{s}} \quad \mathrm{s}_{3}=\mathrm{z}_{\mathrm{s}}{ }^{\prime} / \mathrm{z}_{\mathrm{s}}
$$

These parameters are calculated for each stent point.

\section{EXPERIMENTS AND RESULTS}

The proposed approach was applied to a set of 5 cases in which the patient's pulmonary artery is suffering from stenosis.

\subsection{Visual results Stent fitting into artery}

For given stent and artery model using steps mentioned in previous section, the stent points were matched to appropriate artery points. Judged by visual inspection, the better initial alignment of the stent before applying the matching algorithm improved the final result of stent fitting. The algorithm was tested on data set of 5 patients and satisfactory results were obtained in each case as shown in Fig.4.
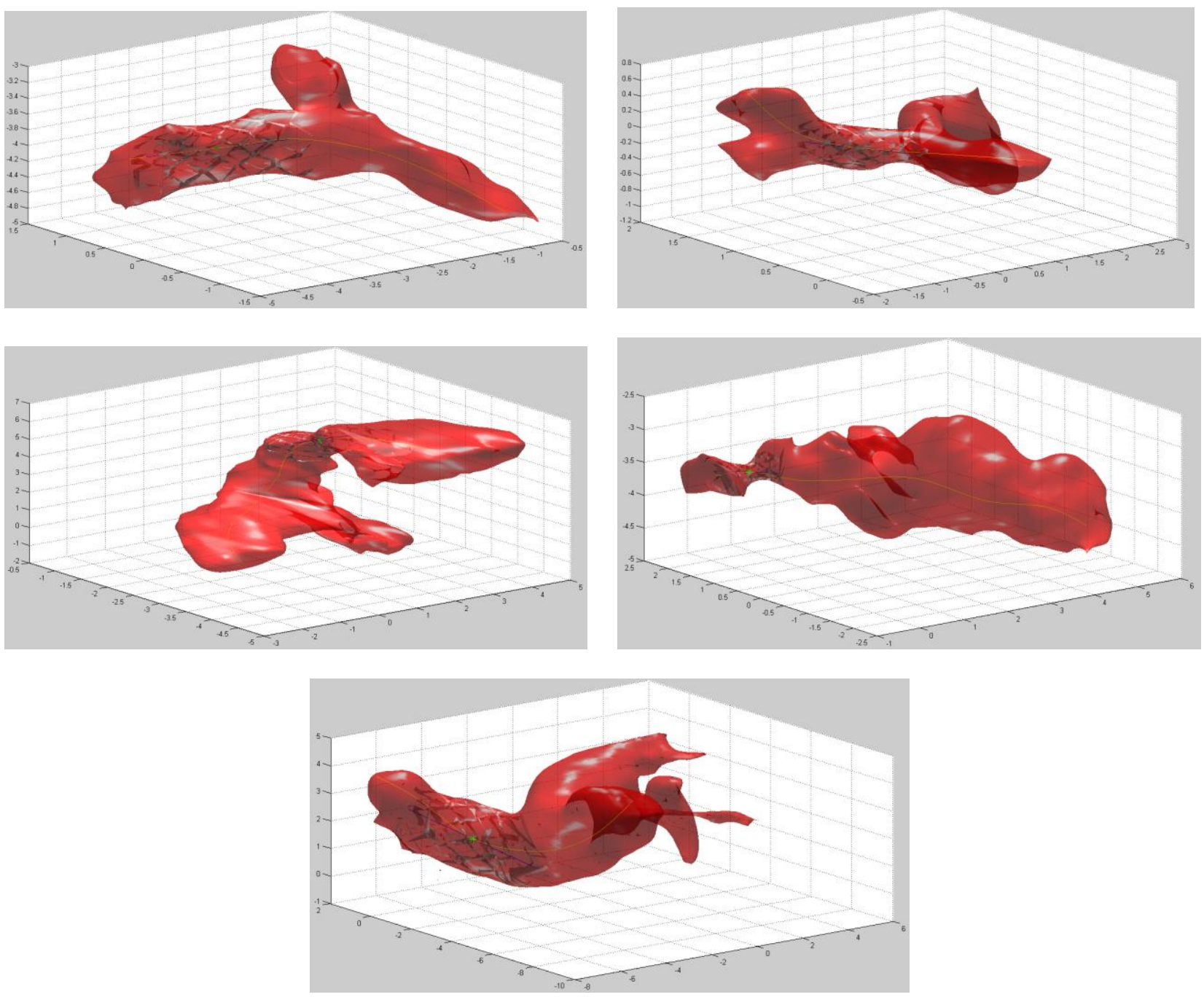

Fig. 4: Stent to artery fitting results as applied on data set of pulmonary artery suffering from stenosis of 5 different patients.

\subsection{Graph of Parameters of CAT Model}

The cylindrical scaling parameters $\left(\mathrm{s}_{1}, \mathrm{~s}_{2}, \mathrm{~s}_{3}\right)$ are calculated for each stent point using step 6 mentioned in previous section. The value of parameters $s_{1}, s_{2}, s_{3}$ indicate change in cylindrical coordinates $\theta_{\mathrm{s}}, \mathrm{r}_{\mathrm{s}}, \mathrm{z}_{\mathrm{s}}$ respectively that each stent point has to go to perfectly fit the artery. The graphs in Fig. 5 indicate value of these parameters for all the stent points. The outliers in the graph $\sim 2 \%$ indicate incorrect matched points.
Table 1 summarizes the values of the cylindrical scaling parameters in terms of the mean and standard deviation calculated for different artery dataset. The mean value of $s_{2}$ multiplied by scaling factor used in Initial Alignment of stent represents the factor by which stent's original size should be changed for perfect fitting of stent. The mean value of other parameters can also be used if greater flexibility is given in designing the stent. 


\section{CONCLUSION AND FUTURE WORK}

In this paper, a method is presented that accurately predicts the stent size done pre-operative. Automatic matching of stent points to artery points was done followed by deformation of the stent which was further modeled using parameters of CAT model. The stent points with exceptionally high value of these parameters indicate the mismatching which was only $2 \%$ revealing high degree of accuracy in stent deformation. However, the running time of the proposed algorithm

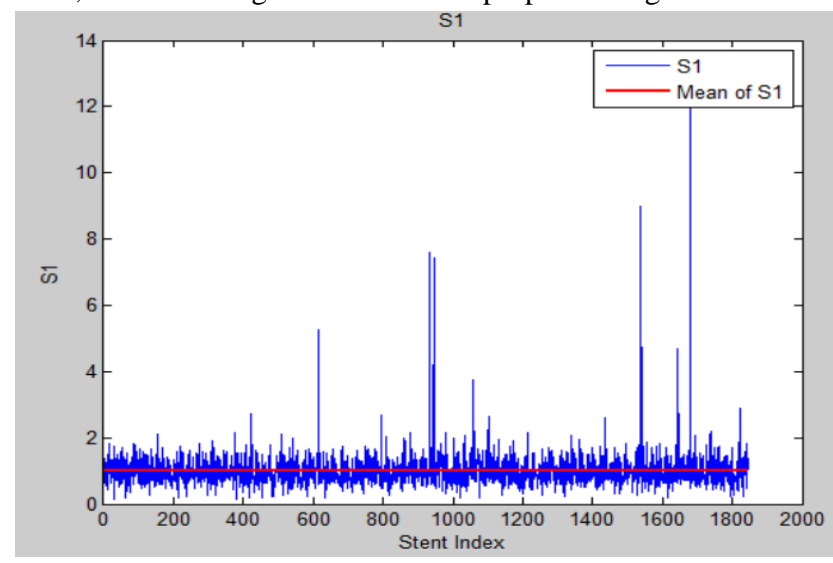

(a) increases as a large artery data set is used. The preprocessing steps in which only relevant artery points are considered instead of entire artery tree should be included in such cases.

In the future, visual simulation of stent expansion inside the artery at the location of stenosis will be done. The parameters of CAT model can be used to control the expansion of the stent depending on degree of stenosis inside the artery vessel. The bio-mechanical properties of pulmonary artery while inserting the stent will be taken into account in future works.

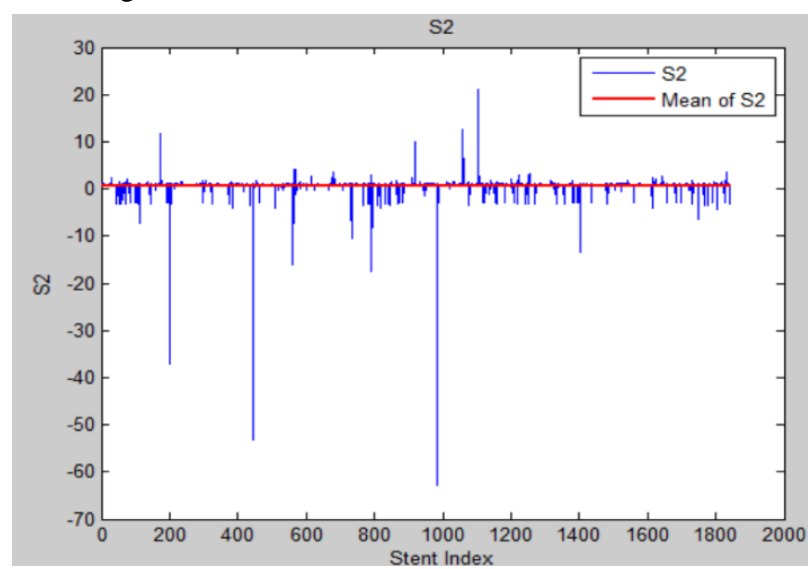

(b)

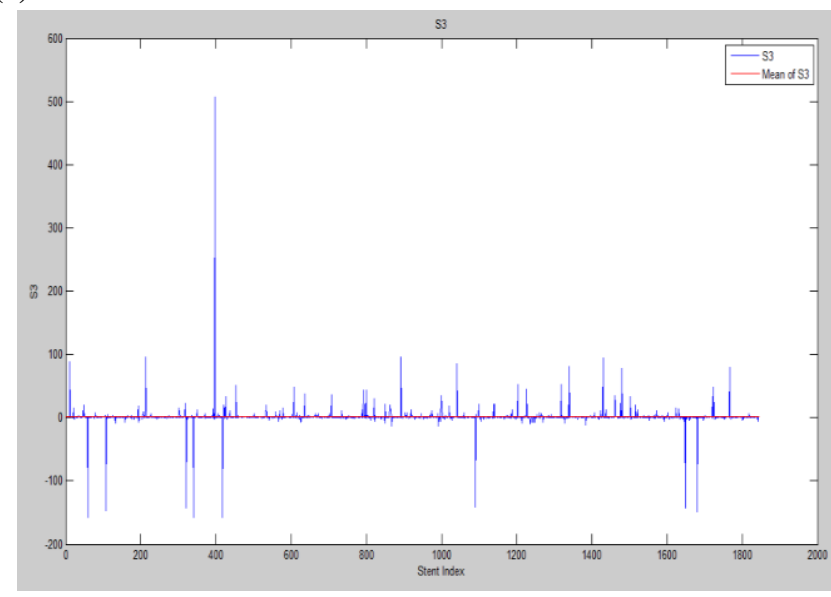

(c)

Fig 5 : Graphs of parameters of Cylindrical Affine Transformation model. (a), (b)and (c) shows values of $s_{1}, s_{2}$ and $s_{3}$ parameters of CAT model.

Table 1: Mean and Standard Deviation of parameters $s_{1}, s_{2}$ and $s_{3}$ of CAT model obtained for various artery data sets.

\begin{tabular}{|c|c|c|c|c|c|c|}
\hline & \multicolumn{2}{|c|}{$\mathrm{s}_{1}$} & \multicolumn{2}{|c|}{$\mathrm{s}_{2}$} & \multicolumn{2}{|c|}{$s_{3}$} \\
\hline & Mean & SD & Mean & SD & Mean & SD \\
\hline Dataset\#1 & 0.6609 & 0.2175 & 0.8023 & 8.9438 & 1.0075 & 0.0258 \\
\hline Dataset\#2 & 0.9872 & 0.5673 & 0.7491 & 2.5106 & 0.9996 & 0.0162 \\
\hline Dataset\#3 & 0.8291 & 0.6340 & 1.6467 & 30.6753 & 1.6066 & 9.5468 \\
\hline Dataset\#4 & 1.1112 & 2.1631 & 0.7021 & 1.3868 & 0.9984 & 0.0192 \\
\hline Dataset\#5 & 0.6701 & 0.2578 & 1.3148 & 8.8139 & 1.6413 & 17.0377 \\
\hline
\end{tabular}

\section{ACKNOWLEDGMENT}

This work was done in collaboration with Sainte-Justine Hospital providing us with patient artery data set needed. We would also like to thank Étienne Vachon and Bruno Roy for their assistance in completing this work. 


\section{REFERENCES}

[1] Rashkind WJ, Miller WW. Creation of an Atrial Septal Defect Without Thoracotomy: A Palliative Approach to Complete Transposition of the Great Arteries. JAMA. 1966;196(11):991-992.

[2] Dotter CT, Judkins MP. Transluminal Treatment of Arteriosclerotic Obstruction. Description of a New Technic and a Preliminary Report of Its Application. Circulation. 1964;30:654-70.

[3] Peters B, Ewert P, Berger F. The role of stents in the treatment of congenital heart disease: Current status and future perspectives. Ann Pediatr Cardiol. 2009;2:3-23.

[4] Balcon R, Beyar R, Chierchia S, et al. Recommendations on stent manufacture, implantation and utilization. Study group of the working group on coronary circulation. Eur Heart $\mathbf{J}$ 1997;18:1536-47.

[5] Serruys PW, de Jaegere P, Kiemeneij F, et al. A comparison of balloon-expandable-stent implantation with balloon angioplasty in patients with coronary artery disease. Benestent study group. N Engl J Med 1994;331:489-95.

[6] Fischman DL, Leon MB, Baim DS, Schatz RA, Savage MP, Penn I, et al. Stent Restenosis Study Investigators. A randomized comparison of coronary-stent placement and balloon angioplasty in the treatment of coronary artery disease. N Engl J Med. 1994;331:496-501.

[7] Macaya C, Serruys PW, Ruygrok P, Suryapranata H, Mast G, Klugmann S, Urban P, den Heijer P, Koch K, Simon R, et al. Continued benefit of coronary stenting versus balloon angioplasty: one-year clinical follow-up of Benestent trial. Benestent Study Group. J Am Coll Cardiol. 1996 Feb;27(2):255-261.

[8] Hendijani N, Manteghi M. Application of singularity expansion method in estimating size of a stent implanted in the artery. Antennas and Propagation (APSURSI), 2011 IEEE International Symposium, 2011 July, pp.131-133.

[9] Jenkins JH, Morrien JP, Bouissaghouane K, Solomon HP.System, method and computer instructions for estimating stent size. EPO Patent EP1700566, filed March 08, 2006, and issued Sept 13, 2006.

[10] Voigt I,Mengue EA, Ionasec R, Mansi T, Georgescu B, Hornegger J, Comaniciu D. Model-based Postoperative Modeling of Stent-Based Devices from CT: Application to TAVI. MICCAI 2012 - Workshop MICCAI-STENT 2012, Nice, France, October 1-5 2012.

[11] Wentzel JJ, Whelan DM, van der Giessen WJ, van Beusekom HM, Andhyiswara I, Serruys PW, Slager CJ, Krams R. Coronary stent implantation changes 3-D vessel geometry and 3-D shear stress distribution. Journal of Biomechanics. 2000;33:1287-1295.

[12] Tu S, Huang Z, Koning G, et al. A novel three-dimensional quantitative coronary angiography system: in vivo comparison with intravascular ultrasound for assessing arterial segment length. Catheter Cardiovasc Interv. 2010;76:291-298

[13] Top A, Hamarneh G, Abugharbieh R. Active Learning for Interactive 3D Image Segmentation. In Medical Image Computing and Computer-Assisted Intervention (MICCAI), volume 6893, pages 603-610, 2011.

[14] Top A, Hamarneh G, Abugharbieh R. Spotlight: Automated Confidence-based User Guidance for Increasing Efficiency in Interactive 3D Image Segmentation. In Medical Image Computing and Computer-Assisted Intervention Workshop on Medical Computer Vision (MICCAI MCV), pages 204213,2010

[15] Tanner C, Carter T, Hawkes DJ, Székely G. Cylindrical affine transformation model for image registration. Proc. SPIE Medical Imaging: Image Processing 2010, vol. 7623, pp. $76232 \mathrm{P}-76232 \mathrm{P}-6$

[16] Weisstein, Eric W. "Point-Line Distance--3-Dimensional." From MathWorld--A Wolfram Web Resource. http://mathworld.wolfram.com/Point-LineDistance3Dimensional.html

[17] de Boor, C., A Practical Guide to Splines, Springer-Verlag, 1978. 